Chapter 13

\title{
Thermoplastic Adhesive for Automotive Applications
}

\author{
Giovanni Belingardi, Valentina Brunella, \\ Brunetto Martorana and Raffaele Ciardiello
}

Additional information is available at the end of the chapter

http://dx.doi.org/10.5772/65168

\begin{abstract}
The objective of this study is to give a general overview on the thermoplastic adhesives used in the automotive sector. Some of the main applications in which the hot-melt adhesives (HMAs) are used in automotive industries are indicated, together with the adhesive characteristics that explain the reasons for their adoption. The chemical and mechanical behavior of these adhesives and the generally used experimental characterization methods are presented and opportunely criticized. In this study, some of the main properties of thermoplastic adhesives are reviewed together with the standard tests used for their characterization. For what concerns the structural performance, single lap joint test is used to determine the shear strength of the adhesive joint. Thermogravimetric analysis and Fourier transform infrared spectroscopy are used to characterize the chemical properties of the adhesive. This study clarifies what are the potentialities of a thermoplastic adhesive in car industries compared to other adhesives.
\end{abstract}

Keywords: thermoplastic adhesive, hot-melt adhesive, mechanical strength, chemical characterization, automotive applications

\section{Introduction}

Adhesives can be categorized in terms of both their chemical composition or polymer type and their physical forms; the latter determines the preferred mode of application and this is the one we refer to in this chapter.

The adhesive joining of different substrates is rapidly diffusing in the manufacturing industries, and nowadays competes with the more traditional techniques of mechanical fastening. 
This is due to several reasons. First, they permit to join parts made of materials that are difficult or even impossible to join in other ways; moreover, they permit the joining of substrates made of different materials (hybrid structures). Other advantages of adhesives include vibration and noise damping, insensitivity to corrosive environment, and direct and indirect reduction in joint weight.

The use of adhesives has progressively increased in the last decades in the automotive industry. The reasons are due to the already cited advantages but in particular for the possibility of making hybrid structures that represent one of the main paths toward lightweight vehicle design. Vehicles have to be designed increasingly lighter with the objective to reduce both their fuel consumption and environmental pollution. In the European Union, particularly, the $\mathrm{CO}_{2}$ limit has been progressively reduced by the European Commission; the short-term normative objective is $95 \mathrm{~g} / \mathrm{km}$ within 2021. In this contest, multimaterial structures and adhesives can offer great contributions to lightweight design.

Adhesives offer a good opportunity of weight reduction [1], in fact, the modern tendency is to replace threaded fasteners and welds with adhesives. Studies show that by reducing $100 \mathrm{~kg}$ of the vehicle weight, the $\mathrm{CO}_{2}$ emission will be reduced by 3-5\% depending on the vehicle size and powertrain type [2].

Further, Chang et al. [3] as well as Belingardi et al. [4] showed that adhesives can improve the stress distribution along the lap zone by a proper design of the joint geometry. Thus, the structural performance can be increased, and in this way, adhesives can offer at least the same stiffness and strength properties than welds. Furthermore, the lightweight trend leads to the design of new vehicle with light aluminum and magnesium alloys, composite materials, and polymers. Therefore, in many of these cases, the adhesive bonding is the only way to make a mechanical connection. Other advantages that can be pursued with the use of adhesives are the already mentioned lack of corrosion if compared to metal fasteners, reduction of noise and vibration, and also the possibility to be functionalized. For instance, adding some properly conceived particles to the base polymer both electrical conductivity and electrical isolation can be enhanced.

Initially, in order to manufacture strong and stiff structural joints thermosetting polymers have been preferred, as they guarantee higher strength and elastic modulus. However, in recent years, the use of thermoplastic adhesives has also been considered and progressively increased in automotive industry, initially for nonstructural applications and then also for structural applications.

Thermoplastic adhesives are plastic materials. The constituents of these adhesives are polymers that melt above a specific characteristic temperature and solidify upon cooling. The thermoplastic adhesive family also belongs to the hot-melt adhesives (HMAs). The HMA is a thermoplastic compound which is applied in the molten state at relatively elevated temperature [5]. The final properties of the adhesive are obtained by natural cooling that usually requires a few seconds. This characteristic of HMA is of particular interest for the automotive industry where the pace of production is very strict. 
The possible constituents of the HMAs can be mixed correctly in order to obtain the required properties. This makes HMAs extremely versatile and various types of HMAs are available in the market.

The adhesive must be applied as a liquid, preferably of low viscosity, both to wet the adherent surface and to flow into the crevices and over the asperities that are universally found in solid surfaces. Usually, HMAs are provided in pellets of different dimensions (from few millimeters to 3-4 cm). In the use of HMA, it is important to mate the two surfaces to be bonded while the adhesive is still fluid. The most used method to spread the HMAs is by means of a hot melt gun.

HMAs are easy to apply. They can be applied by rolling, spreading with a gun, or with an extruder. For this reason, they are used in the manufacturing industry for a lot of different applications, such as:

- Fabrication of luggage, cartons, and trays

- Bookbinding

- Fabrication of furniture

- Fabrication of carpets

- Fabrication of bags, shoes, and other fashion pieces

The above-listed applications are only few of the very big number of applications in which HMAs are used. Since HMAs are generally considered nonstructural adhesives, their main use is limited to applications which do not require high level of adhesion strength. Accordingly, luggage, trays, cartons, and bookbinding industries are the main ones that commonly use HMAs. In other cases, in which higher levels of adhesion strength are required, industries use just a little percentage of HMAs, preferring other types of adhesives, such as the thermosetting ones. In furniture construction, for instance, only $11 \%$ of the total adhesives used are HMAs [6].

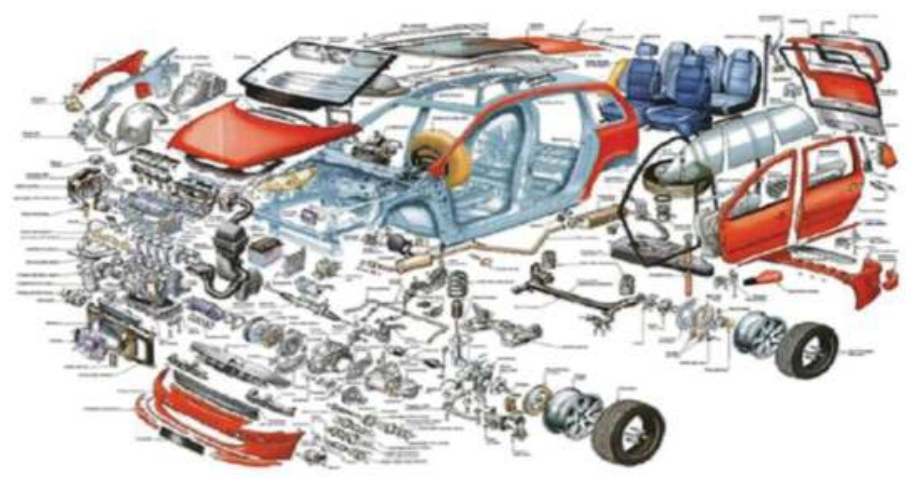

Figure 1. Exploded view of all car components. 
However, in the last decades, due to their versatility, shortness of manufacturing time (compatible with the desired production pace), and good level of adhesion strength, the use of HMAs, but in general for all the adhesives, is also increasing in the automotive industry. The assembly process of the different parts that constitute a vehicle plays an important role in the vehicle construction. In this context, the adhesives represent a good way to simplify the vehicle assembly, in comparison with more traditional fasteners and welding. Figure 1 shows an exploded view of all the vehicle components. This view helps to understand the grade of simplification introduced by the use of adhesives in the vehicle assembly process. For example, the possibility to join all the components of the door panel trims has a high impact on the reduction of cost and on the cycle time of a vehicle.

Nowadays, HMAs are used both for components inside the passenger compartment (generally called "interiors") and for exterior parts. Typical interior components, where HMAs are used to fix, are plastic components such as trim panels, instrument gauge springs, ducts and pipes for air conditioning, sun visor, and many other applications that require the fastening of nonstructural plastic, wooden, and fabric parts. For the exterior components, the environment, where the vehicle has to operate, imposes that the adhesives should overcome the possible degradation that can be consequent to the exposure to severe conditions and, in some cases, also relatively high loading conditions. Typical exterior applications for HMAs are plastic bumpers, back doors, car plates, external air conditioning ducts, lamps, lamp housings, car skirts, and glass roofs. Especially for external parts, the durability and the aging behavior of the HMAs are critical points that need to be assessed in a proper way.

As far as manufacturing process is concerned, HMAs represent a good opportunity to reduce the cycle time. In fact, they can be easily spread by a robotic gun and the solidification of this kind of adhesive occurs in few seconds. During this short time, adhesive reaches a high percentage of the maximum value of its final strength. HMA is also preferred over other adhesive for joining, repairing, and replacing plastic components.

However, HMAs have some disadvantages compared to traditional structural adhesives [7]. In fact, when compared to thermosetting adhesives, which are cross-linked polymers, they exhibit lower heat (i.e., lower operative maximum temperature) and strength resistance.

For what concerns the composition, the HMAs have a main polymer constituent which represents the backbone of the adhesive. The other constituents are generally intended to contribute to modify the main properties of the adhesive in order to increase the affinity with adherent materials and to lower the costs. The base of the HMAs corresponds to polyamides, polyurethane polyesters, EVA (ethylene co-vinyl acetate copolymers), and polyolefin [8]. Polyolefin-based HMAs are used in automotive industries both for interior and exterior components. Applications in the bumper subsystem, door panels, overhead system, seat subsystem, package trays, and instrument panels are only some of the typical uses of HMAs.

HMAs present different constituents, opportunely balanced, in order to give the best performance for the application they are prepared for. In the most typical composition polyethylene and polypropylene are blended together and then combined with a small amount of additives, such as tackifier resins and waxes. 
This work provides a short resume on the types of HMAs used in the automotive industries in order to give a general overview on these kinds of adhesives. The chemical and mechanical behaviors of these adhesives are presented, together with the generally used experimental characterization methods. Some of the main applications in which the HMAs are used in the automotive industries are indicated, together with the adhesive characteristics that explain the reasons for their adoption. This study clarifies what are the potentialities of a thermoplastic adhesive in car industries compared to other type of adhesives.

\section{Materials}

A thermoplastic polymer that is heated to obtain a liquid of flowable viscosity, and, after application, cooled to obtain a solid is termed a hot-melt adhesive (HMA). Although many of these adhesives are polymers of reasonable molecular weight, it is a common practice to incorporate low-molecular weight additives for increasing the fluidity at the manufacturing application temperatures, and to modify properties and lower costs.

So a thermoplastic polymer and a diluent system represent the main composition of an HMA. The thermoplastic polymer constitutes the backbone of the adhesive, providing strength and toughness. The diluent system, represented by antioxidants, fillers, plasticizers, wax, and other resins, opportunely designed, contributes to enhance the specific properties of the adhesive. In this paragraph, the single contributions of the thermoplastic polymers and of the diluent system are discussed.

\subsection{Polymers}

The most popular HMAs are based on polyethylene, polyolefin or their mixtures, ethylenevinyl acetate copolymers (EVAs), polyamides, polyurethane, and polyesters. These constituents need to be precisely designed in order to obtain the desired performance.

Polyethylene (PE) and other polyolefin (PO), such as polypropylene (PP), are adopted in the compound used with plastic materials that are difficult to bond. In general, it is possible to state that the molecular weight of the polymer determines the mechanical properties of the polymers [9]. These kinds of polymers are widely used in automotive applications. The use of this polymers in the HMAs contribute to give higher chemical resistance against acids, bases, and alcohols, good resistance to moisture, and a huge open time, compared to the EVA-based adhesive. The open time is the time between the laying of the adhesive and its solidification. It can be also defined as the time necessary to make the joint in order and to get its bonding quality. When this time is gone, i.e., the adhesive is solidified, it is not possible to constitute a joint. What makes the polyolefin-based HMAs of particular interest for the automotive sector is the high adhesion that this adhesive gives with polypropylene substrates, which is so common in automotive applications.

EVAs are used mainly for low-performance bonding [10-15]. This is one of the most used polymers for the HMAs because of its low cost and large production volume (over 100 million 
tons in 2006) [16]. The use of this polymer in a thermoplastic compound is limited to a maximum temperature of $80^{\circ} \mathrm{C}$, and this polymer has the best mechanical strength between 30 and $50^{\circ} \mathrm{C}$. Since EVA is a copolymer, the balance between the single constituent polymers gives different properties to the compound. In particular, high concentration of ethylene improves the adhesion to nonpolar materials, such as polyethylene, and gives benefit to the mechanical strength. On the other hand, an increase in the vinyl acetate concentration gives benefit to the adhesion with polar materials, such as paper. The prevalence of the vinyl acetate provides higher flexibility and better performance at lower temperatures [17].

Polyamides (PA) are produced in various molecular-weight ranges with consequent variation in melt viscosity, modulus, and softening point that range from 100 to $200^{\circ} \mathrm{C}$ [18]. Open times for these HMAs are typically in the range from 15 to $90 \mathrm{~s}$. Blending of these resins is frequently practiced to obtain the desired properties. These HMAs offer high performance in severe chemical environment. They can bond many different substrates such as those made with metals, wood, ABS, and treated polyethylene and polypropylene.

Polyesters are very similar to polyamides. These polymers are used in the adhesives which require high performance. They have a high application temperature but lower strength and melting point. On the other hand, they present higher resistance to moisture. Polyesters are widely employed to bond fabric.

Polyurethanes promote good adhesion to different surfaces. They have good flexibility at low temperature, because of their low glass transition temperature. These adhesives have a lower application temperature, of the order of $50-70^{\circ} \mathrm{C}$, compared to the others HMAs.

\subsection{Tackifiers}

The presence of tackifiers in the HMA formulation is essentially aimed to increase the adhesive strength between substrates. This is possible because tackifiers are able to increase the surface wetting of the substrates by reducing the viscosity of the adhesive. In addition to the chemical and mechanical characteristics, in most cases, they also give color to the adhesive. The selection of the tackifier involves the cost and the chemical stability of this constituent [6]. Typically, tackifiers have low molecular weight compared to the polymers. The glass transition and the melting temperature are usually above the room temperature. This constituent provides the desired viscoelastic properties to the adhesives. The tackifiers can be divided into three different groups: hydrocarbon resins, rosin esters, and waxes.

\subsubsection{Hydrocarbon resins}

Hydrocarbon resins are a sort of thermal plasticizer. Mainly they are C5 and C9 aromatic hydrocarbon resins. They have a low molecular weight. These resins are a product of petroleum cracking.

C5 aliphatic resins are byproduct of naphtha cracking. They are composed of diolefins, isoprene monomers, and olefins. These kinds of resins confer good heat stability properties and contribute to improve the aging of the adhesive. 
C9 aromatic resins result from gasoline cracking and from the production of ethylene and propylene. Although these resins confer good adhesion strength, they undermine the heat stability and the aging properties [6].

\subsubsection{Rosin esters}

Rosin esters can be produced synthetically or naturally. Rosin resins come from pine tree and they are the oldest raw material that adhesive industries have used. There are three different kinds of rosin resins: gum rosin, wood rosin, and tall oil rosin. Nowadays, rosin esters are widely used because they provide compatibility for a huge variety of adhesive constituents.

The oldest are gum rosins. They are oleoresin (pine gum) of the living pine tree. The collection of resin takes place by wounding of tree and collecting the resin by cups.

Wood rosin is a different oleoresin. It is obtained through the burial of the pine tree stump. After about 10 years in the ground, the bark and sapwood degrade and leave the ground reach of this oleoresin.

Tall oil resin is obtained by industrial distillation of crude oil. Crude oil is composed of about $80 \%$ of acid and tall oil resin. The main characteristic of the tall oil resin is its tendency to crystallize. The use of gum and wood oil resin is preferred to tall oil resin because the last one contains some sulfur contaminants [19].

\subsubsection{Waxes}

The use of waxes in the HMA is mainly intended to reduce the viscosity of the hot melt compound but it is used also to lower the price of the adhesive. The waxes influence not only the viscosity but also other properties such as the softening point, blocking characteristic, and open time. The most used waxes in HMAs are microcrystalline waxes, synthetic waxes, and paraffin waxes. The first and the second ones are used in order to increase the adhesive strength. They contribute also to increase the chemical and mechanical behavior at high temperatures [20]. On the other hand, the paraffin waxes are used to increase antiblocking and barrier properties. The percentage of waxes in HMAs is generally about $20-30 \%$. This quite high value is also consequent to the aim of reducing the adhesive cost [6].

\section{Methods}

In this section we give an overview of the main chemical and mechanical tests used to characterize HMA in the automotive industries. Of course, these characterization tests are applicable also in other research and industrial fields. Here, we explain which are the main targets of each test and how they are conducted. An example of an HMA currently being used by a carmaker is presented too. 


\subsection{The Fourier transform infrared spectroscopy (FTIR) analysis}

The Fourier transform infrared spectroscopy is one of the most popular physical methods for the characterization and identification of polymers and of all the constituents including additives, such as stabilizers, filler plasticizers, and so on. In fact, FTIR spectra provide a pattern of frequencies corresponding to the "fingerprint" of the molecule.

The interferogram (intensity versus frequency) represents the output signal of this analysis [21-23]. The output diagram reports the material transmittance (or absorbance) as a function of the wave numbers (reciprocal of wavelength). This technique is also used to evaluate what is the degradation level of a material, by comparing the spectra after and before the aging [24].

Figure 2 shows the typical functional groups that are possible to identify in FTIR spectrum. The graph is presented as a function of wave numbers in $\mathrm{cm}^{-1}$. As it is possible to see from the figure, in the left part we can find the single bond groups, in particular $\mathrm{N}-\mathrm{H}$ and $\mathrm{O}-\mathrm{H}$ which are located between 4000 and $3200 \mathrm{~cm}^{-1}$. In the range between 3200 and $2800 \mathrm{~cm}^{-1}$ it is possible to find the C-H group. Triple bond groups are in the range between 2300 and $2100 \mathrm{~cm}^{-1}$, while double bond groups range between 1800 and $1500 \mathrm{~cm}^{-1}$. The right part of the spectra is called the fingerprint of the material. Every different compound has a different fingerprint and for this reason FTIR can be used to identify a compound [23].

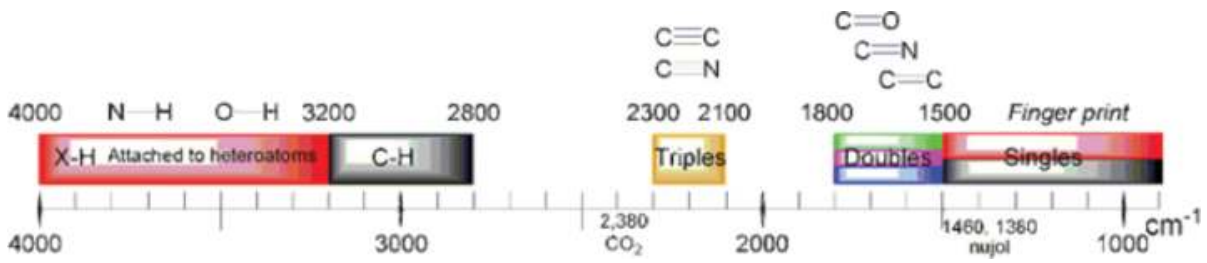

Figure 2. Typical functional groups of the Fourier transform infrared spectroscopy.

\subsection{Thermal analysis}

Thermal analysis embraces all methods in which measurements are made of a property that changes as the temperature changes

\subsubsection{The differential scanning calorimetry (DSC) analysis}

The differential scanning calorimetry (DSC) analysis is used to obtain the thermal behavior, thermodynamic data, and the phase transition temperatures of the considered material (in our case the hot-melt adhesive). With this technique the difference of the heat necessary to rise the temperature of a sample and a reference sample is measured.

The cell environment is purged usually with nitrogen gas, but sometimes it is also interesting to consider different atmosphere (air or oxygen). The test specimen is usually exposed to a heating-cooling-heating treatment cycle within a fixed temperature range at a fixed heating 
rate. During the test, the heat flow is continuously measured as a function of the temperature [25].

\subsubsection{The thermogravimetric analysis (TGA)}

The thermogravimetric analysis is a technique in which the mass of the sample is recorded continuously while the temperature is increased at a constant rate. As the temperature increases, the constituents of the sample start to decompose causing a loss of weight until the complete degradation of the sample.

The TGA instrumentation needs very precise components. These components typically consist of a controlled furnace, a precision balance (able to measure very small mass changes), and a thermocouple. The experiment can be conducted in nitrogen to know thermal stability or in air atmosphere to know the stability during thermal oxidation process.

\subsection{Mechanical properties}

\subsubsection{The single lap joint (SLJ) test}

The mechanical properties of the adhesives are evaluated using different types of joint specimen that have been designed to evaluate specific characteristics. The single lap joint (SLJ) test consists of the tensile test of a specimen made of two substrate parts joined by the adhesive layer of interest (see the specimen sketch in Figure 3). The adhesive layer is mainly submitted to shear loading. This is one of the most used tests in the automotive industry to assess the mechanical properties of the adhesive. This kind of joint is easy to make and it is able to evaluate the adhesive joint strength in its strongest direction (the adhesive layer is loaded in shear). The effect of eventual working temperature, different from the usual room temperature, can be evaluated and can be used with different substrate materials and thickness [26]. Figure 3 shows a sketch of the assembly device that can be used to prepare SLJ specimens under controlled conditions, and a typical testing machine with a configuration of the test. The assembly device, Figure 3(a), allows to fabricate SLJ specimens in the correct way, i.e., it is able to position properly the substrates and to regulate the thickness of the adhesive layer of the specimen. Usually, depending on the adhesive type, some weights are applied on the joint in order to allow the squeezing of the adhesive in excess. This process guarantees that the adherent surfaces are wet enough and in this way it is possible to obtain a great efficiency of the joint. Figure 3(b) shows the testing machine and the joint specimen in its fixtured condition. It can be noted that the intrinsic misalignment of the SLJ can be avoided using tabs at the extremities of the specimens.

The output of this test is the force-displacement or the average shear stress-displacement curve. This test is regulated by ASTM D1002, ASTM D3163, and ASTM D3164 standards that give general specifications for all types of adhesives and adherents. According to these regulations, SLJ is used to determine the shear strength of the adhesive. However, it should be reminded that, as it is well known in the literature, the shear stress distribution along the adherent overlap length is not uniform. This distribution is characterized by two relevant peaks at the two 
overlap extremities. Peaks values depend on several factors such as material modulus, overlapping length, and adhesive layer thickness. Thus, generally, from the performed test only the average value of the shear strength is evaluated.

(a)

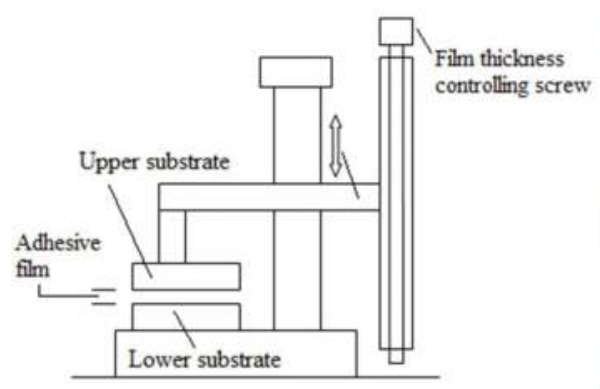

(b)

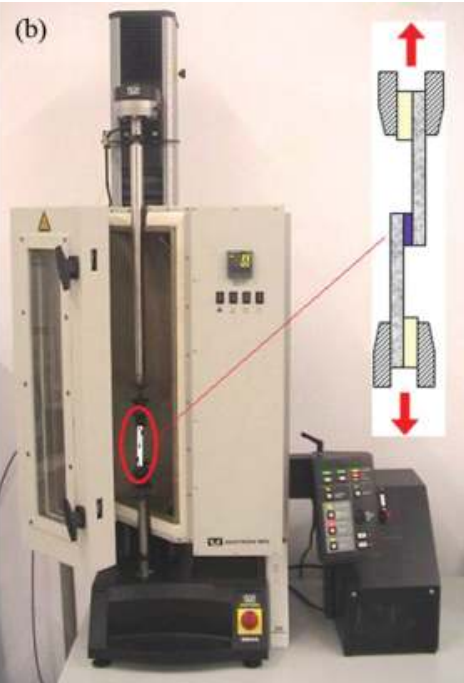

Figure 3. Experimental set-up: (a) assembly device for SLJ preparation, (b) uniaxial tensile testing machine, Instron 5544, with temperature chamber [7].

For what concerns the mechanical behavior, an important analysis for the tested mechanical structures, adhesive joints included, is their failure mode. In the case of adhesives, the analysis of the failure mode is extremely important to verify if the considered adhesive fits with the intended application and to understand the mechanical results. Visual inspection by means of an optical microscope, or a more precise equipment, is able to assess the failure mode. Figure 4 shows the typical failures of adhesive-bonded structures. There are three base ways in which SLJ may fail:

a. Cohesive failure

b. Adhesive failure

c. Substrate failure

In the first one, the failure occurs in the adhesive layer, as shown in Figure 4(a). In this case, it is possible to find the clear presence of the adhesive material on both the two adherent faces [7]. The second failure mode presents a complete separation between the adhesive layer and one of the substrates, as shown in Figure 4(b). It should be mentioned that mixed modes also, i.e., with the presence of both adhesive and cohesive failures in the same joint, can be encountered. The last failure mode presents a failure in one of the two adherents, as shown in Figure 4(c). Figure 4(d) shows a typical adhesive tensile failure of a HMA joint. 


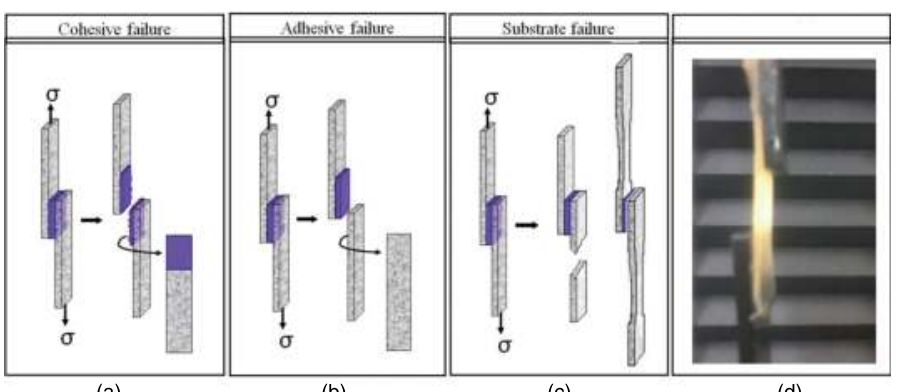

(a)

(b)

(c)

(d)

Figure 4. Failure modes of SLJ specimen [7]: a) cohesive failure, b) adhesive failure, c) substrate failure, d) adhesive tensile failure.

\section{Results for one HMA sample experimental characterization}

In this section, the chemical and mechanical characteristics of one typical HMA commonly used for interior and exterior automotive applications are presented. Polypropylene substrates have been used for the mechanical test (SLJ). First, the reason for the use of HMA is because of the good performance this adhesive gives with polypropylene substrates. The use of polypropylene in car components has increased over the time because of its low cost and good mechanical performance. Furthermore, both of them are easy to recycle and give the possibility to reduce the weight of the component in comparison to other plastic materials [27]. Another important characteristic of this particular adhesive is the possibility to apply without any surface treatment. As mentioned in the introduction, this adhesive is sold in a granular form. These pellets are melted in a portable furnace and sprayed on the components by a gun. This procedure fits perfectly with the typical manufacturing process of the automotive industries. In fact, this system can be easily mounted on a robot arm and thus adapted to perform joining process for different subassemblies.

\subsection{Characterization of the hot hot-melt adhesive}

First of all, we report the main results of the typical chemical-physical characterization process for an HMA used in the automotive field. In this section, FTIR, TGA, and DSC test results as well as the main result of the SLJ mechanical characterization tests are presented. The objective is to give a complete example of the procedure together with the main characteristics of a typical adhesive. These results are essential to understand whether the adhesive fits with the peculiar application requirements.

\subsubsection{FTIR}

Figure 5 shows FTIR spectrum of a commonly used HMA. It is possible to evidence the presence of the $\mathrm{CH}_{2}$ and $\mathrm{CH}_{3}$ group between the values of 2800 and $3000 \mathrm{~cm}^{-1}$ on the wave 
number axis, with a stretching peak at $2916 \mathrm{~cm}^{-1}$. In this range, these are the highest peaks of the spectrum. Two other peaks are observed in the range between 1459 and $1376 \mathrm{~cm}^{-1}$ that can be interpreted as deformation of $\mathrm{C}-\mathrm{H}$ links in $\mathrm{CH}_{2}$ and $\mathrm{CH}_{3}$ groups. Other absorption peaks are visible with lower wave number and lower intensities that are related to isotactic polypropylene.

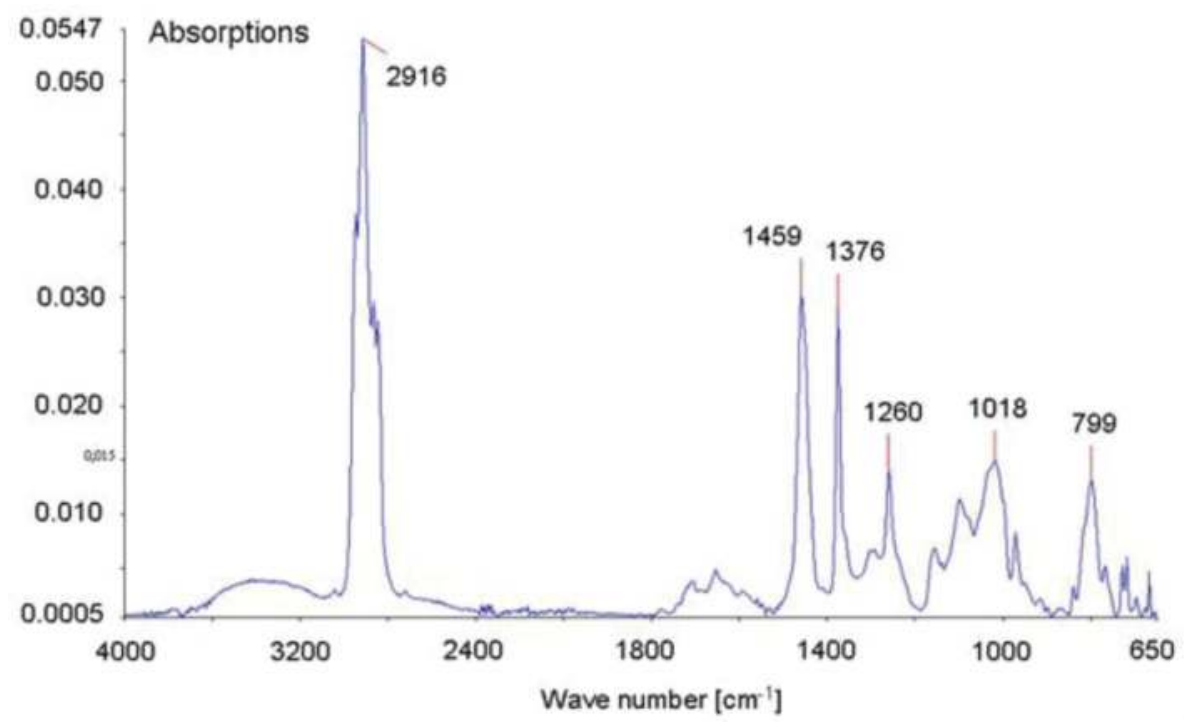

Figure 5. FT-IR spectrum for a polyolefin-based hot-melt adhesive [7].

This spectrum also shows two peaks at 1163 and $971 \mathrm{~cm}^{-1}$, which are related to amorphous polypropylene. Moreover, other peaks related to crystalline polypropylene, in particular, the peaks at 997 and $838 \mathrm{~cm}^{-1}[28,29]$, are visible. Polyethylene absorbance peaks at 720 and 730 $\mathrm{cm}^{-1}$ are presented [25]. More specifically, the peak at $720 \mathrm{~cm}^{-1}$ is related to both crystalline and amorphous polyethylene, while the peak at $730 \mathrm{~cm}^{-1}$ is the peak of the crystalline phase of the PE. The presence of the two phases of PE means that the copolymer has long chains of PE that can be crystallized.

The other region of interest in this spectrum is the region between 1800 and $1600 \mathrm{~cm}^{-1}$. In this interval, the peak at $1778 \mathrm{~cm}^{-1}$ could be related to the presence of a partially hydrolyzed anhydride group. Another interesting peak is at $1710 \mathrm{~cm}^{-1}$ that refers to carbonyl acid $\mathrm{C}=\mathrm{O}$. The presence of these groups could be due to a treatment used in order to increase the polarity and consequently the adhesive properties of the HMA.

The recognition of these components and groups in the FTIR spectrum shows the potentiality of this analysis. The spectrum is able to show that this adhesive is a copolymer PP-PE HMA and the knowledge of the different groups is important to find the affinity with different adherents. This analysis can be also extremely useful to assess the aging of the HMA. For what 
concerns the studied adhesive, when the adhesive is aged, it oxidizes, especially in the ranges between 1800 and $1500 \mathrm{~cm}^{-1}$. This behavior can be recognized by the presence of higher peaks in the cited range [23].

\subsubsection{TGA results}

Figure 6 shows the result of the TGA analysis with the chosen HMA. The green curve is relative to the degradation of the HMA with the increase of the temperature in nitrogen atmosphere. The thermal degradation of the polyolefin-based HMA takes place in one step. This behavior is typical of the polyethylene and polypropylene polymers that are the backbone of this adhesive. Degradation in inert atmosphere starts at about $250^{\circ} \mathrm{C}$ and ends almost at $480^{\circ} \mathrm{C}$ with a maximum degradation rate at $451^{\circ} \mathrm{C}$. Higher temperatures do not cause any further degradation process. The carbon residue of the HMA is $0.68 \%$ of the original sample weight.

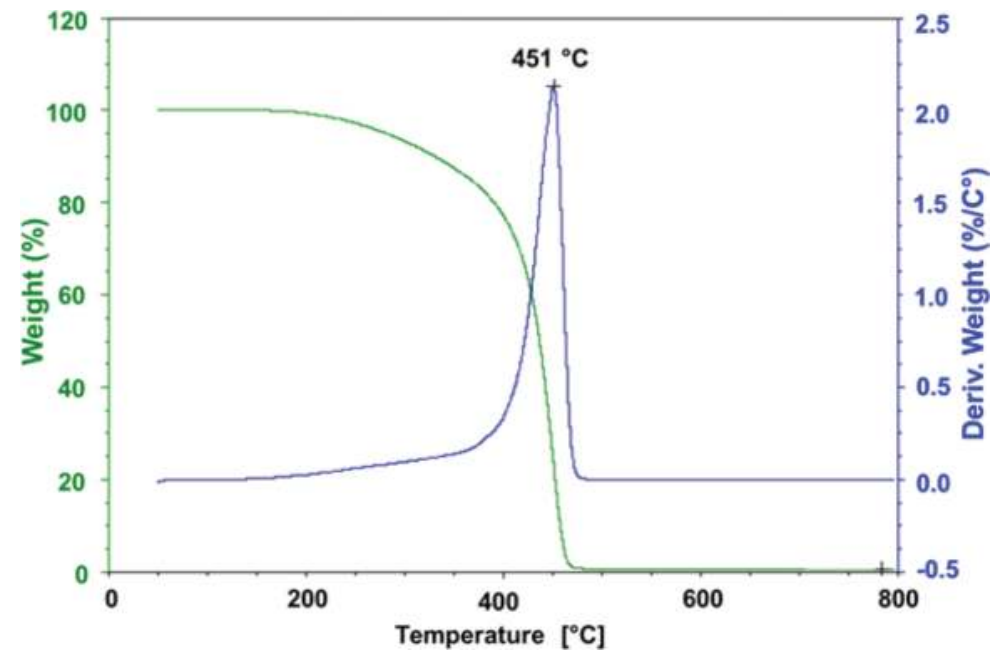

Figure 6. TGA result for a polyolefin-based hot-melt adhesive [7].

The blue curve, in the same figure, shows the derivative of the mass loss as a function of the temperature. This is the weight loss velocity and it is extremely significant to capture the processes that cause little variation of the mass (i.e., loss of volatile and nonvolatile constituents) and to evidence the temperature at which the degradation rate is maximum.

\subsubsection{DSC}

Figure 7 reports the relevant part of the DSC result curve. As it can clearly be seen in Figure 7, the considered polyolefin-based HMA exhibits a sharp phase change peak with a combination of shallow and broad peaks. In the heating curve, the endothermic peaks are located at about 124 and $154^{\circ} \mathrm{C}$ and could be associated to the melting point of polyethylene and 
polypropylene, respectively. In fact, as for FTIR results, a crystalline phase of PE and a crystalline phase of PP are present in HMAs.

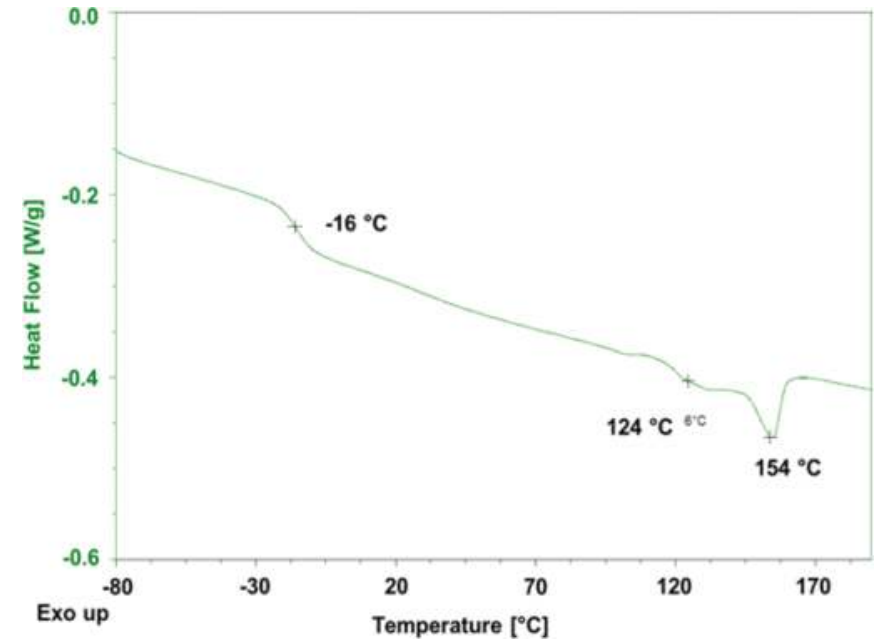

Figure 7. DSC results for a polyolefin-based hot-melt adhesive [7].

As it can be seen in Figure 7, only one glass transition temperature is exhibited at $\left(-16^{\circ} \mathrm{C}\right)$ and this is correlated to a copolymer PE/PP. This means that the polyolefin-based HMA is more susceptible to become brittle and easily loses its toughness at or below this temperature.

\subsubsection{SLJ tests}

The main information about the modality of single lap joint test execution has been presented in a previous section. In addition, now it has to be specified that the test velocity for the results presented in this section is $100 \mathrm{~mm} / \mathrm{min}$. Figure $\mathbf{8}$ shows the typical diagram of the applied load, displacement curves (Figure 8a), and the average shear stress, displacement curves (Figure 8b), in the case of specimen width of $20 \mathrm{~mm}$, overlap length between the two substrates of $12.5 \mathrm{~mm}$ and three different values of the thickness of the adhesive layer, as specified in the diagram legend.

It is well visible that the first part of the curve is linear, and then there is a smooth passage to a nonlinear behavior that is connected to the plastic deformation of the adhesive layer. The plasticization starts from the overlap extremities, where, as mentioned, the stresses have their maximum values and move toward the overlap midspan. Then the maximum (peak or failure) load is reached and the sustained load decrease quite sharply.

The figure gives the possibility to make a comparison of the curves corresponding to three different values of the adhesive layer thickness, which is one important factor. It is well visible that, in accordance with the literature $[30,31]$, the smaller the adhesive layer thickness the larger the SLJ strength. 


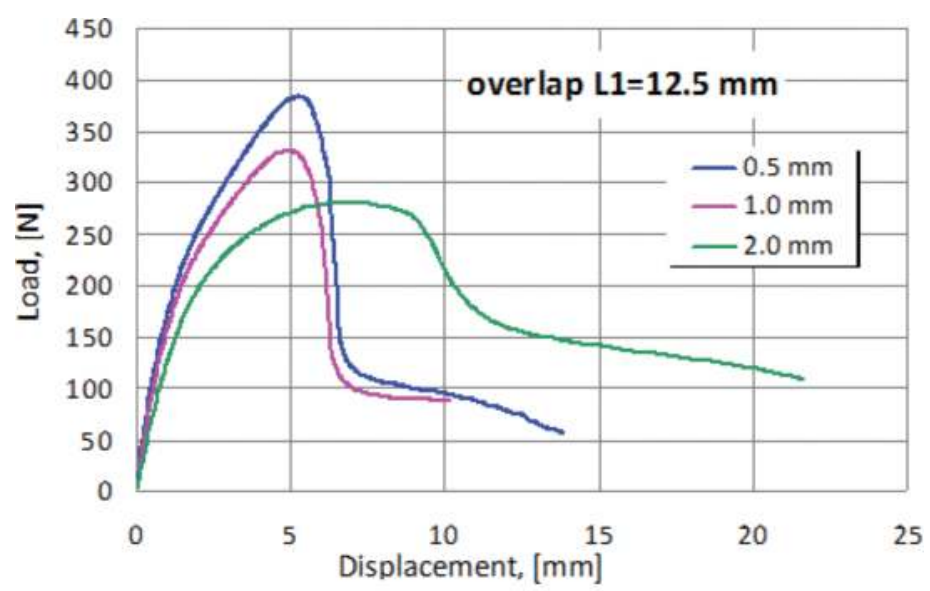

a)

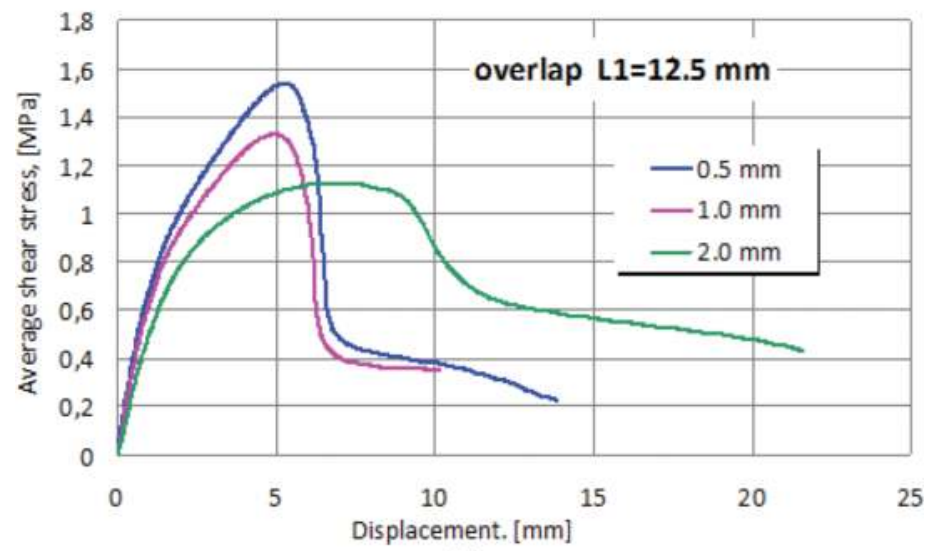

b)

Figure 8. Typical force displacement (a) and average shear stress-displacement curve (b) obtained with SLJ tests for the considered HMA.

As anticipated in Section 1, HMAs are generally not considered structural adhesives. The mechanical characteristics of the polyolefin HMA are reported in this section. Having done several SLJ tests similar to that reported in Figure 8, by changing the overlap length, it is possible to develop an analysis of the effect of the two considered main factors (the overlap length and the adhesive layer thickness) on the structural performance. The reported results show the dependency of the failure loads of the SLJ test on different overlap lengths and adhesive thickness. Figure 9 shows that larger adhesive layer thickness generally leads to lower failure loads, while longer overlap leads to higher failure loads. As it can be noticed, differently from the other two considered overlap lengths, the specimens with the overlap of $50 \mathrm{~mm}$ have the same failure loads. The reason for this has to be found in the different failure mode. In these latter cases, the failure zones are localized in the substrates and not in the adhesive layer. 


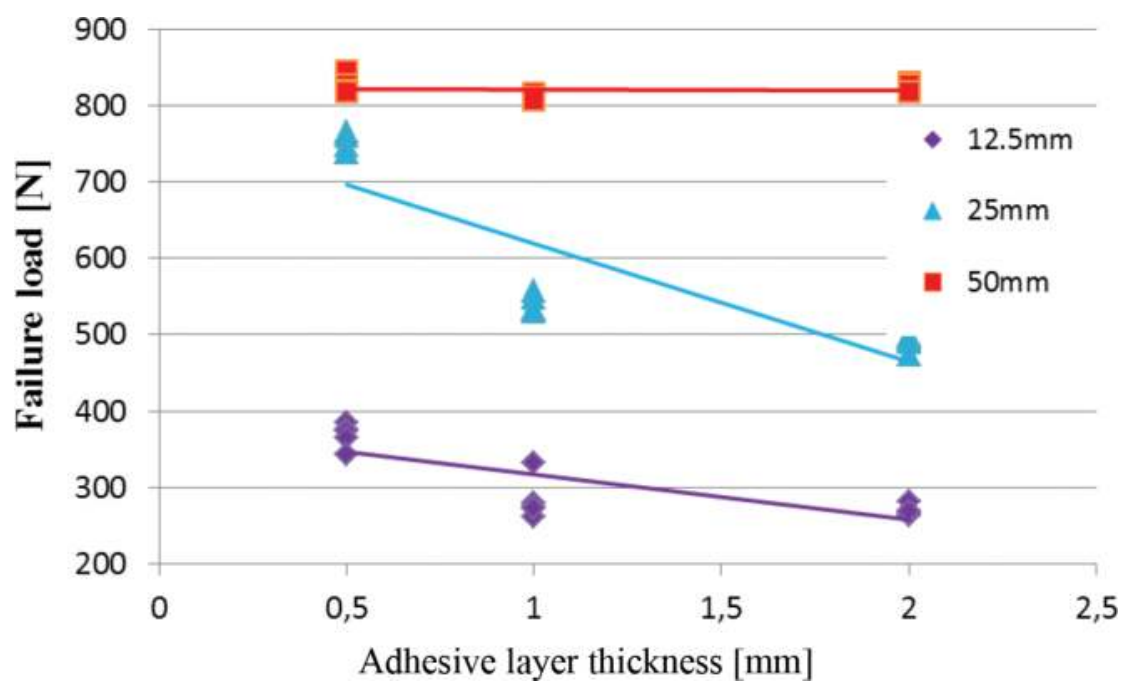

Figure 9. Failure load results from SLJ test for a Polyolefin based hot melt adhesive [21].

Figure 10 shows the same results but in terms of the average shear strength of the SLJ test, calculated by rating the failure load to the adhesion area. In this case both values and descending trends are very similar, if we look the overlap length of 12.5 and $25 \mathrm{~mm}$, while the curve is once again flat for the $50 \mathrm{~mm}$ overlap. This last trend is different for the abovementioned reason.

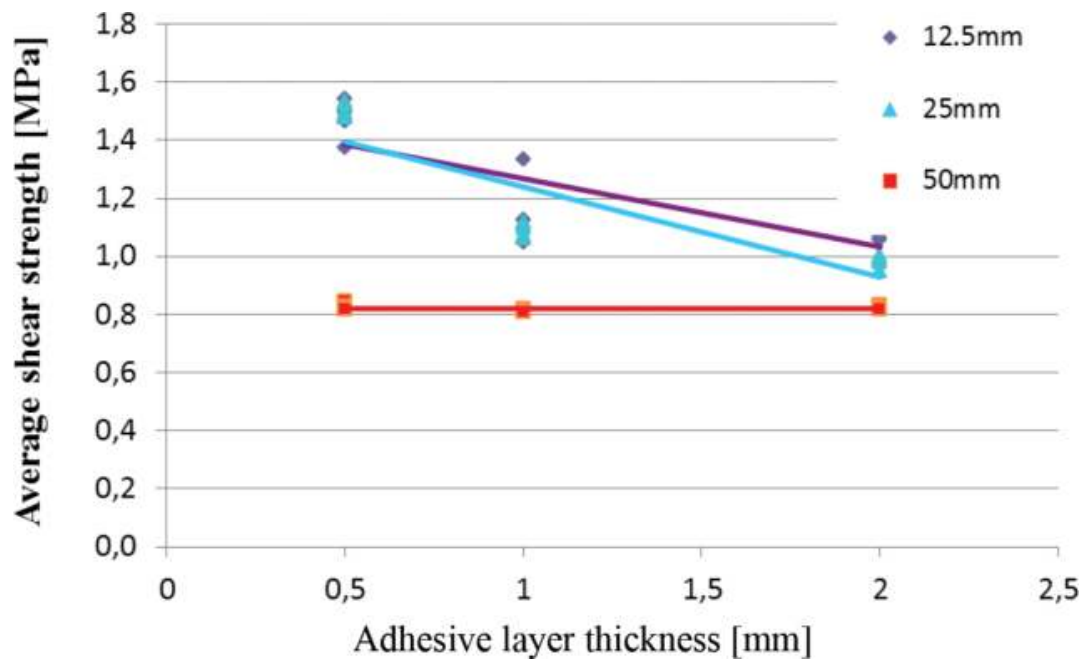

Figure 10. Average shear strength results from SLJ test for a Polyolefin based hot melt adhesive [21]. 
Visual inspection shows that the most dominant failure type is cohesive failure for overlap length of up to $25 \mathrm{~mm}$, as it can be seen in Figure 11. Indeed, for the 0.5 and $1 \mathrm{~mm}$ adhesive layer thickness, $12.5 \mathrm{~mm}$ overlap SLJ exhibits cohesive failure and equivalent shear strength to $25 \mathrm{~mm}$ overlap sample. For $2 \mathrm{~mm}$ adhesive layer thickness, the observed dominant failure type is adhesive failure at one tip followed by adhesive layer shear failure. Finally, the $50 \mathrm{~mm}$ overlap length specimens show insignificant failure load variation with adhesive layer thickness and generally exhibit substrate failure.

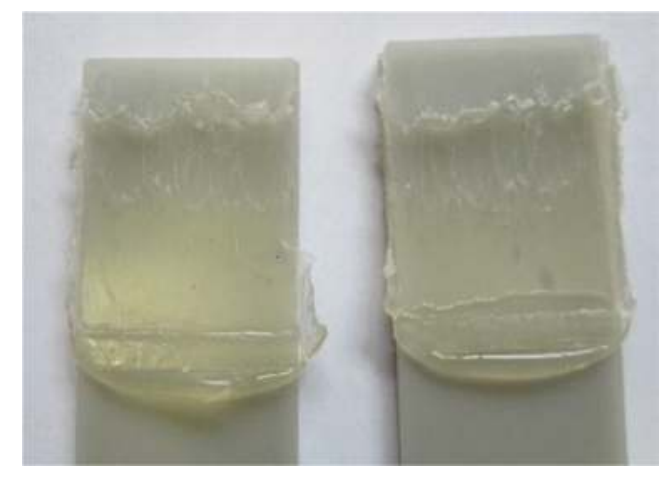

Figure 11. Failure surface of a SLJ test, overlap length $25 \mathrm{~mm}$, adhesive thickness $1 \mathrm{~mm}$.

The subsequent investigation of the load-displacement diagrams of the tested specimens leads to a better understanding of the substrate contribution and of the SLJ response to the type of failure mode for given geometry changes. The load-displacement curves expected from the SLJ shear tests are different in the case of 12.5 and $25 \mathrm{~mm}$ overlap length specimens with respect to $50 \mathrm{~mm}$ ones. This expectation is confirmed by the diagrams that are shown in Figure 10. The diagrams in Figures 12(a) and (b) have been obtained with two different values of the substrate overlap lengths and have similar trends, as previously described commenting Figure 9. On the contrary, curves in Figure 12(c) have completely different trends. After the elastic phase, a maximum is reached quite rapidly and then the curves have a slow decrease while the displacement reaches quite large values. For this latter type of specimens, the dominant plastic deformation of the PP substrate is responsible for this wide range of displacement after yielding. It is of interest to observe the curves reported in Figure $\mathbf{1 3}$ where one of the characteristic curves of the SLJ and the tensile curve of the PP are superimposed. The SLJ curve is just a little bit below the PP curve, confirming that the structural behavior of this joint is dominated by the substrate (as depicted in Figure 12(c)). Figure 12(d) collects in one diagram the average shear stress-displacement curves for the three overlap lengths and one particular value of the adhesive layer thickness $(0.5 \mathrm{~mm})$. The shear stress curves for the two lower overlap lengths have similar trends and values. The shear stress-displacement curve for $50 \mathrm{~mm}$ overlap samples exhibits a completely different trend but, as explained before, this is related to the fact that it does not represent the actual adhesive curve, but the consequences of the PP substrate deformation. 


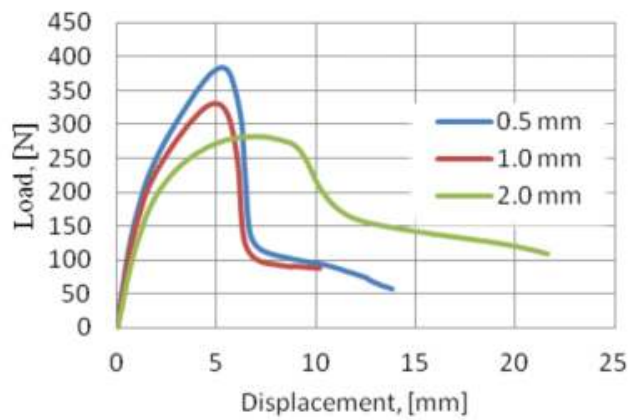

a) $\mathrm{L} 1=12.5 \mathrm{~mm}$

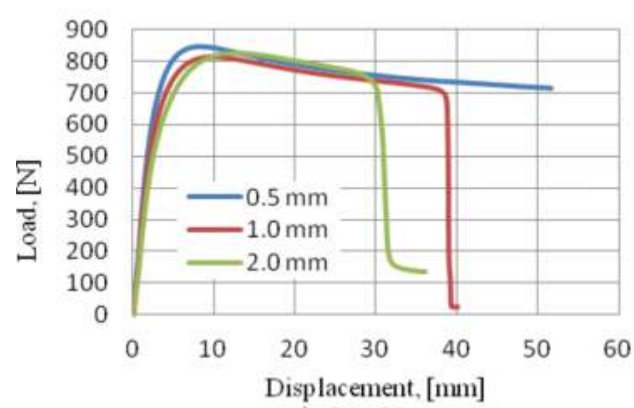

c) $\mathrm{L} 3=50 \mathrm{~mm}$

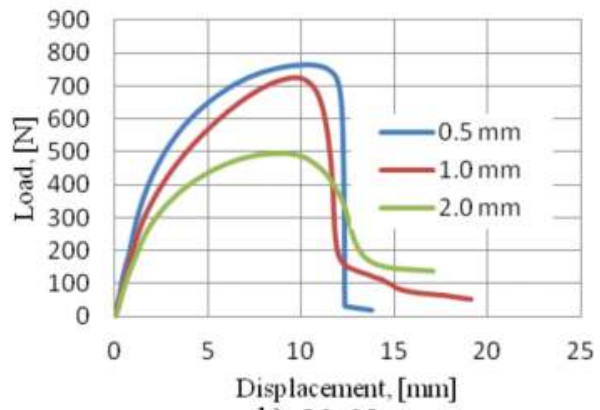

b) $\mathrm{L} 2=25 \mathrm{~mm}$

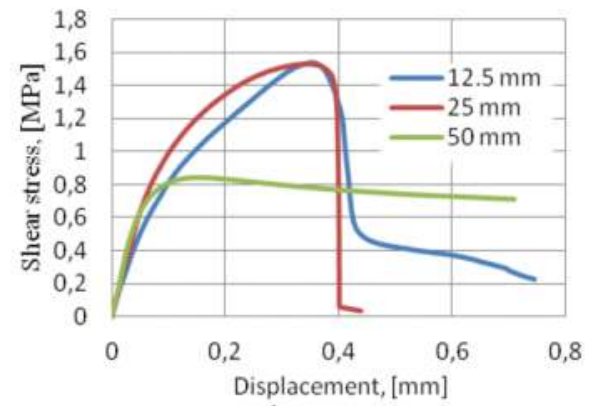

d) $\mathrm{T}=0.5 \mathrm{~mm}$

Figure 12. $(\mathrm{a}-\mathrm{c})$ Load versus displacement curves of SLJ for different bond lengths and adhesive layer thickness. (d) Average shear stress versus displacement curve [7].
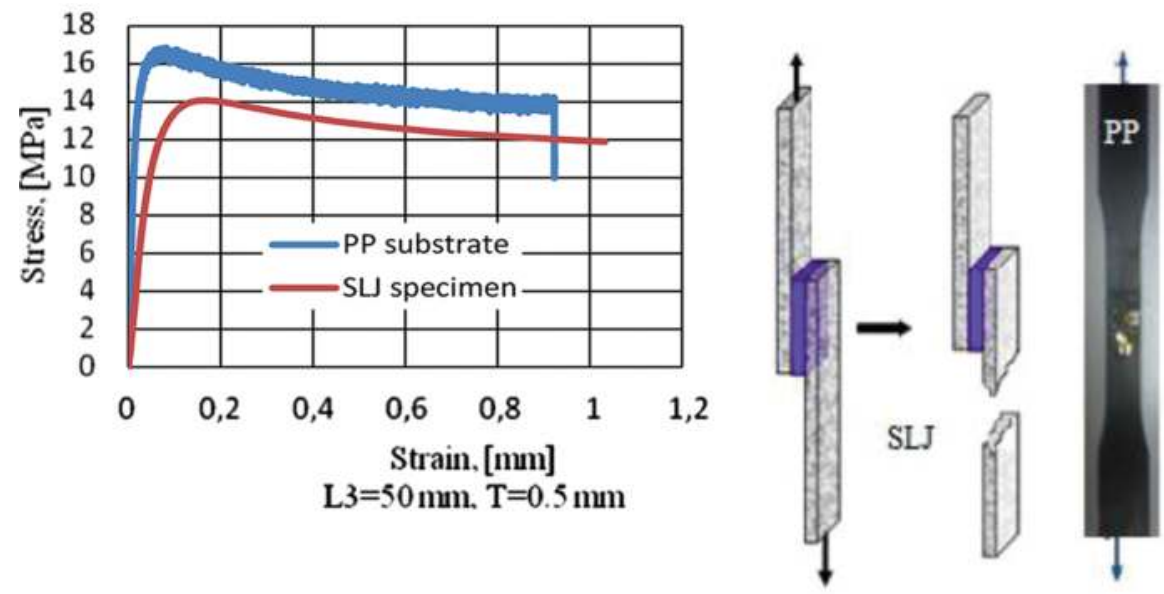

Figure 13. left - Typical stress-strain curve for SLJ and PP substrate test [7]; right - substrate failure during SLJ tensile test. 
Finally, in order to explain the differences that are visible between the two curves reported in Figure 13, it is important to remember that the SLJ test introduces a slight but not negligible bending moment in the substrates. Due to the eccentricity of the applied load and uneven stress distribution near the joint extremities, the substrate experiences higher stress values than those induced by simple tensile tests.

\section{Conclusion}

This work represents a general overview of the current use of HMAs in the automotive industries. It also gives a comprehensive systematic summary of the ways adopted to characterize from the chemical-physical and mechanical points of view of an HMA. HMAs are a wide class of flexible thermoplastic adhesives, which can be employed for a wide range of uses both in the automotive field and in other mechanical applications. The motivations of this work are essentially due to the attempt of covering the lack of systematic information on this topic.

Some of the most relevant applications adopted by many carmakers have been listed. The adoption of HMAs has been progressively increased due to their particular properties: better recyclability, good mechanical performance, low cost, good resistance to moisture, good chemical stability, and lack of curing time. HMAs exhibit a lower strength and heat resistance when compared with structural thermosetting adhesive. Even though HMAs are traditionally associated with nonstructural applications (e.g., packaging, sealing, and clothing industries) in many literatures, the properties, that these materials give to the single lap joint loaded in shear, has resulted in an expansion of their use also in structural applications, such as in automotive vehicles, as well as in other nonstructural applications, such as furniture.

The results of some experimental tests have been reported, in relation to PP substrates, and duly commented, putting in evidence the role of two main factors: the overlap length and the adhesive layer thickness, in determining the strength of the SLJ. The comparison with the failure modes show that HMAs exhibit high plastic elongation compared to other structural adhesive (such as thermosetting adhesive), which generally present a brittle behavior.

\section{Author details}

Giovanni Belingardi ${ }^{*}$, Valentina Brunella ${ }^{2}$, Brunetto Martorana ${ }^{3}$ and Raffaele Ciardiello ${ }^{1}$

*Address all correspondence to: giovanni.belingardi@polito.it

1 Politecnico di Torino, Torino, Italy

2 Università di Torino, Torino, Italy

3 Centro Ricerche Fiat S.C.p.A., Torino, Italy 


\section{References}

[1] Mallik P. Materials, Design and Manufacturing for Lightweight Vehicles. Woodhead publishing, Sawston, Cambridge (UK); 2010. 384 p. ISBN: 978-1845697822

[2] Structural bonding of lightweight cars [Internet]. 2010. Available from: http:// www.dowautomotive.com/pdfs/Structural_Adhesives_Brochure.pdf [Accessed: 2016-06-01].

[3] Chang B, Shi Y, Dong S. Comparative studies on stresses in weld-bonded, spot-welded and adhesive-bonded joints. Journal of Materials Processing Technology. 1999; 87:230 236. DOI: 10.1016/S0924-0136(98)00355-0

[4] Belingardi G, Chiandussi G. Stress flow in thin walled box beams obtained by adhesive bonding joining technology. International Journal of Adhesion \& Adhesives. 2004; 24:423-439. DOI:10.1016/j.ijadhadh.2003.11.009

[5] Pizzi A, Mittal K. Handbook of Adhesive Technology. $2^{\text {nd }}$ ed. Marcel Dekker, New York (US), 2003, 999 p. ISBN: 0-8247-0986-1

[6] Skeist I. Handbook of adhesives. $3^{\text {rd }}$ Van Nostrand Reinhold, New York (US). 1989; 931 p. ISBN: 978-0442276348

[7] Koricho E, Verna E, Belingardi G, Martorana B, Brunella V. Parametric study of hotmelt adhesive under accelerated ageing for automotive applications. International Journal of Adhesion and Adhesives. 2016; 68:164-181. DOI: 10.1016/j.ijadhadh. 2016.03.006

[8] Shin H,Hamed G. Peel adhesion and viscoelasticity of poly (ethylene-co-vinyl acetate)based hotmelt adhesives. The influence of wax. Journal of Applied Polymer Science. 1997; 63:333-342. DOI: 10.1002/(SICI)1097-4628(19970118)63:3<333::AID-APP8>3.0.CO; $2-\mathrm{O}$

[9] Su W. Principles of polymer design and synthesis. Springer, Berlin (D). 2013;306 p. DOI: 10.1007/978-3-642-38730-2

[10] Park Y, Joo H, Kim H, Lee Y. Adhesion and rheological properties of EVA-based hotmelt adhesives. International Journal of Adhesion \& Adhesives. 2006; 22:571-576. DOI: 10.1016/j.ijadhadh.2005.09.004

[11] Kinloch A. Adhesion and adhesives-science and technology. Chapman and Hall, London (UK); 1994; 440 p. DOI: 10.1007/978-94-015-7764-9

[12] Petrie E. Reactive hot-melt adhesives for better structural bonding. Metal Finishing. 2008; 106:39-43. DOI: 10.1016/S0026-0576(08)80125-0

[13] Shin H, Hamed G. Peel adhesion and viscoelasticity of poly (ethylene-co-vinyl acetate)-based hot-melt adhesives. I. The effect of tackifier compatibility. Journal of 
Applied Polymer Science. 1997; 63:323-331. DOI: 10.1002/(SICI)1097-4628(19970118)63:3<323::AID-APP7>3.0.CO;2-P

[14] Li W, Bouzidi L, Narine S. Current research and development status and prospect of hot-melt adhesives: a review. Industrial \& Engineering Chemical Research. 2008; 47:7524-7532. DOI: 10.1021/ie800189b

[15] Park Y, Kim H. Hot-melt adhesive properties of EVA/aromatic hydrocarbon resin blend. International Journal of Adhesion \& Adhesives. 2003; 23:383-392. DOI: 10.1016/ S0143-7496(03)00069-1

[16] Anon. Production: grow is the norm. Chemical Engineering News. 2008; 84:59-68. DOI: 10.1021/cen-v084n028.p059

[17] Finley T. Secure devices for personal or article identification, such as driver's licenses and vehicle license plates, and methods of producing such improved devices. 2016. U.S. Patent 9272496.

[18] Frihart C. Specific adhesion model for bonding hot-melt polyamides to vinyl. International Journal of Adhesion \& Adhesives. 2004; 24:415-422. DOI: 10.1016/j.ijadhadh. 2003.11.008

[19] The chemistry of tackifying resins. [Internet] 1990. Available from: https:// www.pstc.org/files/public/Donker.pdf [Accessed: 06-05-2016].

[20] Using Waxes in Hot Melt Adhesives. [Internet] 2009. Available from: http://adhesives.specialchem.com/tech-library/article/using-waxes-in-hot-melt-adhesives [Accessed: 06-05-2016].

[21] Colthup N, Doly L, Wiberley S. Introduction to infrared and Raman spectroscopy. $3^{\text {rd }}$ ed. Academic Press Limited, London (UK); 1990. 547 p. ISBN: 978-0121825546

[22] Hummel D. Baum A. Atlas of Polymer and Plastic Analysis. Carl Hanser Verlag GmbH \& Co., Munich (D), 1998. 1250 p. ISBN: 978-3446161269

[23] Ciardiello R, Belingardi G, Martorana B, Fondacaro D, Brunella V. A study of physical and mechanical properties of a nano-modified thermoplastic adhesive in normal and accelerated ageing conditions. European Conference on Composite Material. (ECCM17); 26-30 June, 2016.

[24] Mazurin O. Problems of compatibility of the values of glass transition temperaturesGlass Physics and Chemistry. 2007; 33:22-36. DOI: 10.1134/S108765960701004X). 2008. 363 p. ISBN: 978-0-8155-1533-3

[25] Ebnesajjad S. Adhesives technology handbook. 2nd ed. William Andrew, Norwich (NYUS). 2008. 363 p. ISBN: 978-0-8155-1533-3

[26] Polypropylene Compounds for Automotive Applications. [Internet] 2010. Available from: http://www.sumitomochem.co.jp/english/rd/report/theses/docs/ 20100100_a2g.pdf [Accessed: 06-05-2016]. 
[27] Park Y, Kim H, Kim H, Rafailovich M, Sokolov J. Viscoelastic properties and lap shear strength of EVA/aromatic hydrocarbon resins as hot-melt adhesives. Journal of Adhesion Science and Technology. 2003; 17:1831-1845. DOI: 10.1163/156856103322538714

[28] Hummel S. Atlas of Polymer and Plastic Analysis. Carl Hanser, Weinheim. 1991; 1250 p. ISBN: 9783527257980

[29] Gallo R, Brambilla L, Castiglioni C, Ipsale S, Severini F, Quasso F, Consolati G. Environmental degradation of a novel ethylene-propylene copolymer in thick sheets. European Polymer Journal. 2005; 41:359-366. DOI: 10.1016/j.eurpolymj.2004.09.014

[30] Adams R, Peppiatt N. Stress analysis of adhesively bonded lap joints. The Journal of Strain Analysis for Engineering Design. 1974; 9:185-196. DOI: 10.1243/03093247V093185

[31] da Silva L, Rodrigues T, Figueiredo M, de Moura M, Chousal J. Effect of adhesive type and thickness on the lap shear strength. The Journal of Adhesion. 2006; 82:1091-1115. DOI:10.1080/00218460600948511 